\title{
The ultra-high energy cosmic rays image of Virgo A
}

\author{
Radomír Šmída* \\ Karlsruhe Institute of Technology, Germany \\ E-mail: radomir.smida@kit.edu
}

\section{Ralph Engel}

Karlsruhe Institute of Technology, Germany

\begin{abstract}
Arrival directions of ultra-high energy cosmic rays from the direction of ten brightest radio sources lying within $50 \mathrm{Mpc}$ from our Galaxy were studied by using recent models of the largescale Galactic magnetic field. A detailed study, where also small-scale turbulent magnetic field component was implemented, is presented for the radiogalaxy Virgo A. This radiogalaxy is located far from the Galactic plane which leads to a unique image of this UHECR source candidate, if the flux is composed from a mixture of intermediate mass nuclei. We present a method suitable for identifying cosmic rays arriving from this close-by radiogalaxy.
\end{abstract}

The 34th International Cosmic Ray Conference,

30 July- 6 August, 2015

The Hague, The Netherlands

${ }^{*}$ Speaker. 


\section{Introduction}

The search of a point source of ultra-high energy cosmic rays (UHECRs), defined as cosmic rays with energy above $50 \mathrm{EeV}$ in this paper, lacks an answer more than half-century after their discovery $[1,2]$. The primary particles are charged nuclei accelerated in astronomical objects. Moreover, the distance to such sources must be within $\sim 100 \mathrm{Mpc}$ for UHECRs because of energyloss processes on various photon backgrounds $[3,4]$.

Categories of astronomical objects powerful enough to accelerate nuclei up to the highest measured energy [5] have been classified in the past [6]. For the search of the site of the UHECR origin an identification of a local point source is of the primary concern, because the flux from such source located within a few tens of Mpc won't be significantly attenuated for most of nuclei [7, 8]. Moreover, extragalactic magnetic fields might be rather uniform over such distance. The most interesting position of the source in the sky is at high Galactic latitude, because the large-scale Galactic magnetic field (GMF) between the source and the Earth has the least complex structure in this direction.

Any nuclei of an electric charge $Z$ will be deflected during their propagation through the GMF. Under an assumption that all particles from the same source travel through the same magnetic field the angular deflection will scale linearly with the electric charge. It is therefore useful to study the angular deflection as a function of the magnetic rigidity $R(\mathrm{~V})=E(\mathrm{eV}) / Z$ instead of energy $E(\mathrm{eV})$.

We will assume that UHECRs arrive to the Galaxy in a parallel beam from an extragalactic point source and information about the source position is not lost during the propagation in the extragalactic space. Under such assumptions we can study an effect of the large-scale GMF and also small-scale turbulent magnetic fields on the source image at the Earth.

\section{Simulations}

Point source candidates and the GMF model must be selected before performing a simulation. For the former the full-sky the catalogue of radiogalaxies of the local universe [10] was selected. This catalogue was prepared for any study of UHECRs and provides information required for our analysis. Ten brightest radiogalaxies within the distance $D=50 \mathrm{Mpc}$ have been selected from this catalogue and their list is provided in Tab. 1. The closest object from our selection is Cen A at the distance 3.6 Mpc followed by Virgo A and Fornax A.

These ten celestial objects are not uniformly distributed across the sky, because they follow the local mass distribution. For example the sky positions of radiogalaxies NGC 5090 and Cen A are separated by only $1^{\circ}$ and in the case of Virgo A and NGC 4261 only $7^{\circ}$.

All simulated particles were randomly generated in a flat circular area having its centre $50 \mathrm{kpc}$ from the Galactic centre. The position of the centre was at the sky position of a radiogalaxy. The area was always perpendicular to the direction connecting the Galactic centre and the radiogalaxy. The radius of this area was $20 \mathrm{kpc}$ and $10 \mathrm{kpc}$ for our simulations without and with the turbulent magnetic field, respectively.

The radius of a detector at the position of the Earth was $100 \mathrm{pc}$. The maximum time to track particle was one million years and the minimum time step was 10 years. The CRT uses adaptive Runge-Kutta integration methods to determine the trajectory of a charged particle through 


\begin{tabular}{cccccc}
\hline Name & $\alpha$ & $\delta$ & $l$ & $b$ & $D$ \\
\hline Fornax A & 50.7 & -37.2 & 240.2 & -56.7 & 20.90 \\
Virgo A & 187.7 & 12.4 & 283.8 & 74.5 & 18.44 \\
NGC 5090 & 200.3 & -43.7 & 308.6 & 18.8 & 46.89 \\
Cen A & 201.4 & -43.0 & 309.5 & 19.4 & 3.59 \\
NGC 4261 & 184.8 & 5.8 & 281.8 & 67.4 & 32.15 \\
NGC 4696 & 192.2 & -41.3 & 302.4 & 21.6 & 41.71 \\
IC 5063 & 313.0 & -57.1 & 340.0 & -38.7 & 46.63 \\
NGC 5793 & 224.9 & -16.7 & 342.0 & 36.3 & 46.86 \\
NGC 2663 & 131.3 & -33.8 & 255.7 & 5.6 & 29.97 \\
NGC 7626 & 350.2 & 8.2 & 87.9 & -48.4 & 47.77 \\
\hline
\end{tabular}

Table 1: Ten brightest radiogalaxies within $D<50 \mathrm{Mpc}$ [10]. Columns: name of radiogalaxy, right ascension, declination, galactic longitude and latitude, distance in Mpc.

a magnetic field according to the relativistic Lorentz force. Detailed description of the CRT code can be found in [9] and in the manual.

\section{Regular GMF}

The Janson\&Farrar (JF12) GMF model [11] was primarily used, but also other models were studied $[12,13]$. We will present only results obtained for the JF12 model in this paper. A parallel beam of particles pointed towards the Earth was generated in a circular area of $20 \mathrm{kpc}$ diameter at the Galactocentric distance $50 \mathrm{kpc}$. The studied magnetic rigidity was between 1 and $150 \mathrm{EV}$ and the spectral index was 1 .

In a uniform magnetic field the angular deflection is inversely proportional to the magnetic rigidity $\theta=K / R$. Results of our simulations above $30 \mathrm{EV}$ were used to obtain the constant $K$. Then all results were compared with the inverse linear fit, see for example Fig. 1 for Virgo A.

Our results are summarised in Tab. 2, where the numerical constant $K$ and the rigidity where simulated arrival direction differs more than $3^{\circ}$ from the fit are provided. The uncertainty of values in Tab. 2 is $\pm 5^{\circ} \mathrm{EV}$ for the constant $K$ and $\pm 1 \mathrm{EV}$ for $R_{3^{\circ}}$. Two values of $R_{3^{\circ}}$ are provided for NGC 2663, because of two different trajectories hitting the Earth at the same rigidity. The same happens for NGC 4696 and NGC 5090, but only below $30 \mathrm{EV}$. (Let us notice, that two possible trajectories at the same rigidity have been found even for Virgo A and other GMF model, namely Sun et al. [12].)

Results obtained for the JF12 model, see Tab. 2, show that the angular deflection is $2^{\circ}-4^{\circ}$ at $R=100 \mathrm{EV}$ for all studied radiogalaxies. The simulated data are described by the inverse linear fit below $10 \mathrm{EV}$ only for radiogalaxies lying far from the Galactic plane, i.e. $|b|>50^{\circ}$. All other radiogalaxies have $R_{3^{\circ}} \geq 10 \mathrm{EV}$, i.e. the energy of 70 and $140 \mathrm{EeV}$ for nitrogen and silicon nuclei, respectively.

Interestingly, the lowest value of $R_{3^{\circ}}$ has the strongest local radiogalaxy Fornax A. However, by checking the sky map one can recognise that even if the angular deflection scales with $R$ the 


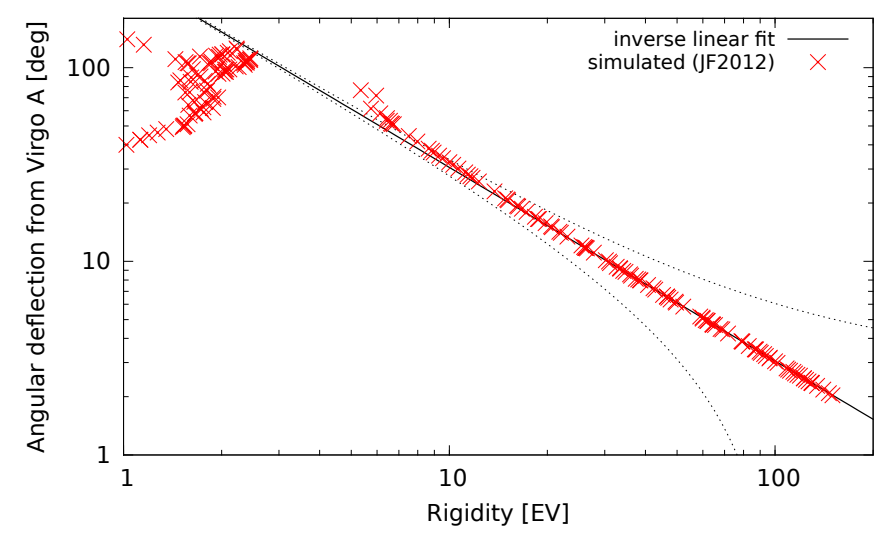

Figure 1: Red crosses show the angular deflection from Virgo A as function of the rigidity, black solid line shows the inverse linear fit and dashed lines the $\pm 3^{\circ}$ offset from the fit. Notice missing successful hits between 2.5 and 5.4 EV, i.e. when particles cross the Galactic plane.

\begin{tabular}{ccc}
\hline Radiogalaxy & $K\left({ }^{\circ} \mathrm{EV}\right)$ & $R_{3^{\circ}}(\mathrm{EV})$ \\
\hline Fornax A & 242 & 4 \\
Virgo A & 306 & 7 \\
NGC 5090 & 183 & 16 \\
Cen A & 191 & 15 \\
NGC 4261 & 274 & 8 \\
NGC 4696 & 149 & 12 \\
IC 5063 & 330 & 10 \\
NGC 5793 & 370 & 14 \\
NGC 2663 & 192 & 10,25 \\
NGC 7626 & 413 & 10 \\
\hline
\end{tabular}

Table 2: Results for the JF12 model of the regular GMF. Parameters of the linear fit described in the text are provided.

direction abruptly turns at $R \simeq 10 \mathrm{EV}$, see Fig. 2 .

It is reasonable to study a location of a point-source only at rigidities, where the information is not completely lost or vastly reduced by the propagation. We can identify this rigidity with $R_{3^{\circ}}$.

In the case of Virgo A the simulated results above $7 \mathrm{EV}$ agree within $3^{\circ}$ with values obtained from the inverse linear fit as can be seen in Fig. 1 and Tab. 2. An increase of discrepancy between calculated and simulated values below $R_{3^{\circ}}$ is caused by more complex structure of the GMF in the vicinity of the Galactic plane. Due to this plane none arrival of simulated particles between 2.5 and 5.4 EV was registered at the Earth and arrival directions of particles at rigidities below $2.5 \mathrm{EV}$ are highly scattered, see Fig. 3. 


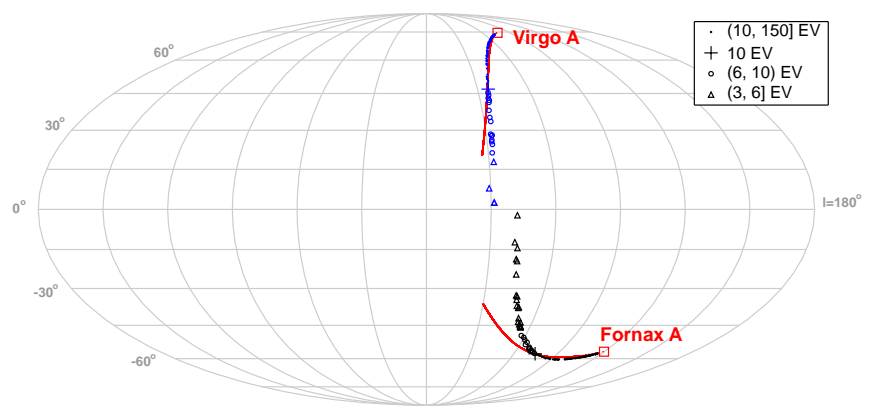

Figure 2: Arrival directions of cosmic rays from Virgo A and Fornax A, indicated by big red squares. The Galactic coordinates are used and the Galactic centre is in the centre of this map. Blue (black) colour is used Virgo A (Fornax A). For clarity only events above 3 EV are shown.

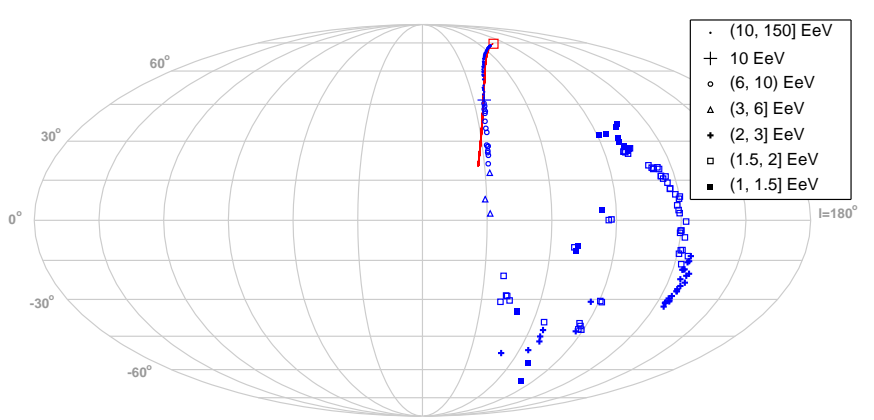

Figure 3: Arrival directions of cosmic rays from Virgo A between 1 and 150 EV. Red line connects sky positions of a radiogalaxy and $10 \mathrm{EV}$ event and its length is $60^{\circ}$.

\section{Turbulent magnetic field}

From ten studied local radiogalaxies the arrival deflection follows a single arc and the deflection can be described by the inverse linear fit below $R=10 \mathrm{EV}$ only for two of them, Virgo A and NGC 4261. For further investigation of the UHECR image we have selected the radiogalaxy Virgo A.

The small-scale random magnetic fields have to be included to the large-scale regular GMF to obtain more realistic description of arrival directions. We used four realisations of random magnetic field characterised by a Kolmogorov spectrum and generated in CRT. Four fields of spheres with sizes and field magnitude drawn from Gaussian distributions and each a randomly oriented field vector were generated. Each random magnetic field realisation is described by eight parameters: number of divisions within the KRF box in each space coordinate axis $(N X, N Y, N Z)$, length of each side of the KRF box ( $L X, L Y, L Z)$, the minimum and maximum wavelengths ( $L M I$ and $L M X)$. Values used in our simulations are in Tab. 3.

As for the regular GMF study a parallel beam of particles was generated in a circle centred at the sky position of Virgo A at the galactocentric distance of $50 \mathrm{kpc}$. The differences from the previous case were as follows: the diameter was $10 \mathrm{kpc}$, the rigidity was $5-150 \mathrm{EV}$ and the 


\begin{tabular}{cccc}
\hline Realisation & $N X, N Y, N Z$ & $L X, L Y, L Z(\mathrm{kpc})$ & $L M I, L M X(\mathrm{pc})$ \\
\hline KRF1 & $256,256,256$ & $5.12,5.12,5.12$ & 5,100 \\
KRF2 & $256,256,256$ & $5.12,5.12,5.12$ & 5,100 \\
KRF3 & $256,256,256$ & $5.12,5.12,5.12$ & 5,512 \\
KRF4 & $256,256,256$ & $2.56,2.56,2.56$ & 5,100 \\
\hline
\end{tabular}

Table 3: Eight parameters describing four magnetic field realisations. Two realisations (KRF1 and KRF2) have the same parameters, but different seed number was used for them.

spectral index was 3. The lower limit of the rigidity was set at $5 \mathrm{EV}$ to avoid trajectories crossing the Galactic plane. Simulations were stopped when at least 90 hits were reached.

The least difference between a KRF realisation and the regular JF12 field was for KRF4, which had the smallest side length among used KRF realisations. Otherwise, all KRF realisations show similar features: small offset at the highest rigidities, departure more than $\pm 3^{\circ}$ from the inverse linear fit between 10 and $20 \mathrm{EV}$ and large scattering of angular deflections between 5 and $8 \mathrm{EV}$. All these effects could be expected and they indicate an increasing importance of the turbulent component of the GMF on the propagation at rigidities below $\sim 10 \mathrm{EV}$.

Our results for Virgo A can be compared with similar study made for Cen A [14], which lies close to the Galactic plane $\left(b=19.4^{\circ}\right)$.

\section{Triangular area}

Arrival directions for four different realisations of the random magnetic field are shown in Fig. 4. Our simulations show that arrival directions of the majority of events form a triangular rather area in all four cases. Similar observation has been presented in [15].

This can be understood by looking in two types of deflection experienced by UHECRs. First, a rigidity-dependent offset from the source position can be expected due to deflections in large-scale magnetic fields. Second, a scattering of the arrival directions caused by turbulent magnetic fields with the amplitude inversely depending on the rigidity is expected for UHECRs from the same source. The combination of these two effects then leads to a triangular rather than circular image in the sky.

We suggest to construct the triangular area from sky positions of a source candidate and one event. These two positions form an axis of the triangular area. The event should lie at angular distance $\theta(1, S)$ from the source candidate larger than an angular resolution of a cosmic-ray observation and an angular deflection expected in the GMF. from the studied source candidate. We will call such event the leading event. The triangular area is then defined by the axis, the maximum angular distance $\psi$ from the source candidate ${ }^{1}$ and two half-opening angles $\alpha_{1}$ and $\alpha_{2}$ along the axis, see Fig. 5.

In Fig. 4 we show the triangular area for four KRF realisations. The same parameters were used for the triangular area in all four cases to allow a comparison of arrival directions between our

\footnotetext{
${ }^{1}$ The value of $\psi$ must be less than $90^{\circ}$ to avoid shrinking of the spherical area at larger angular distances.
} 


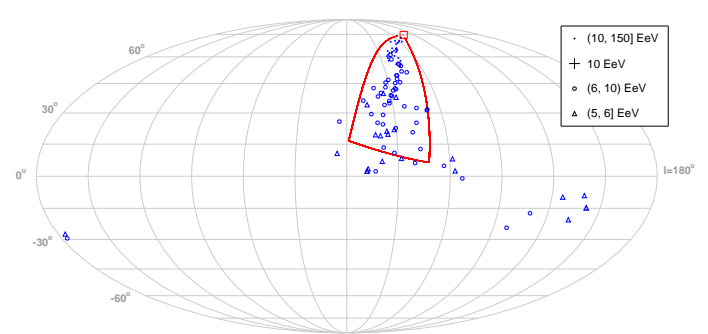

(a) JF12 + KRF1

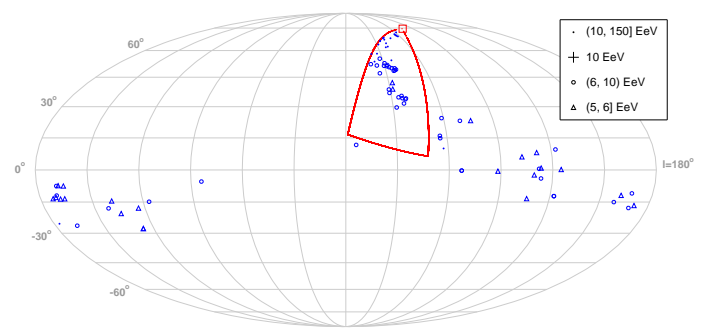

(c) JF12 + KRF3

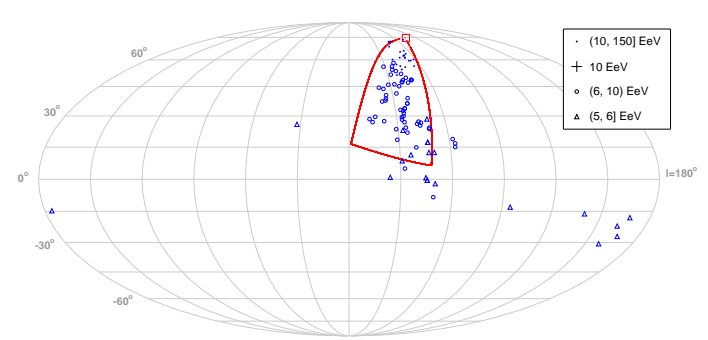

(b) JF12 + KRF2

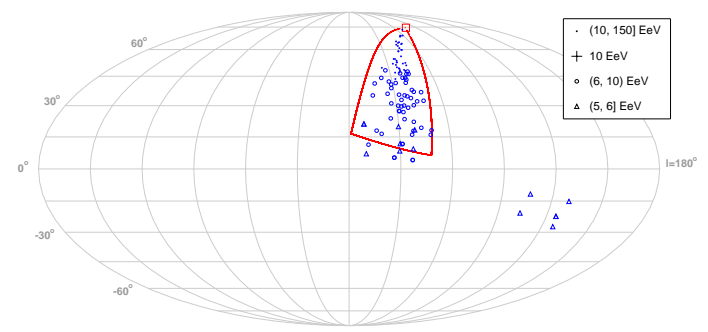

(d) JF12 + KRF4

Figure 4: Arrival directions for a combination of the JF12 large-scale regular GMF field and four random field realisations. The triangular area is also shown.

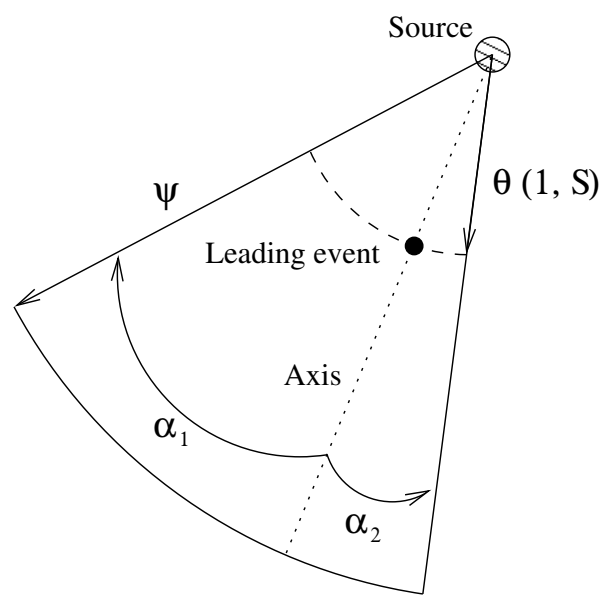

Figure 5: Sketch of the triangular area described by angles $\psi, \alpha_{1}$ and $\alpha_{2}$. The point source and leading event are separated by the angular distance $\theta(1, S)$.

simulations. The parameters are as follows: the leading event is $10 \mathrm{EV}$ event from the simulation for the JF model with only regular magnetic field, $\psi=70^{\circ}, \alpha_{1}=\alpha_{2}=25^{\circ}$.

\section{Conclusions}

Angular deflections in the GMF for the 10 brightest radiogalaxies located within the distance of $50 \mathrm{Mpc}$ were studied. From them Virgo A was investigated more extensively and even an effect 
of various turbulent magnetic fields was included.

We have shown that the expected UHECR image of Virgo A has an asymmetric shape for the magnetic rigidity above $5 \mathrm{EV}$. If the UHECR flux is a mixture of nuclei (i.e. protons and heavy nuclei) the image takes a triangular rather than circular shape. This unique feature is due to high galactic latitude of Virgo A and can be used for an identification of Virgo A as the UHECR point source.

Our analysis is based on the JF12 model and even if it is the most elaborated model of the GMF, it does not provide complete picture of magnetic fields neither in the interstellar space nor in the Galactic halo. Any discrepancy between this GMF model and real environment may affect the arrival directions, particularly at low rigidities. Nevertheless, our results can be adopted to any GMF model and cosmic-ray data.

\section{Acknowledgement}

It is a pleasure to acknowledge discussions with our colleagues of the Pierre Auger Collaboration.

\section{References}

[1] J. Linsley et al., Phys. Rev. Lett. 6 (1961) 485

[2] J. Linsley, Phys. Rev. Lett. 10 (1963) 146

[3] K. Greisen, Phys. Rev. Lett. 16 (1966) 748

[4] G.T. Zatsepin, V.A. Kuzmin, JETPL 4 (1966) 78

[5] D.J. Bird et al., Astrophys. J. 441 (1995) 144

[6] A.M. Hillas, Annu. Rev. Astro. Astrophys. 22 (1984) 425

[7] K. Kotera \& A. Olinto, Annu. Rev. Astro. Astrophys. 49 (2011) 119

[8] D. Allard, Astropart. Phys. 39-40 (2012) 33

[9] M.S. Sutherland et al., Astropart. Phys. 34 (2010) 198

[10] S. van Velzen et al., Astron. \& Astrophys. 544 (2012) A18

[11] R. Jansson \& G.R. Farrar, Astrophys. J. 757 (2012) 14

[12] X.H. Sun et al., Astron. \& Astrophys. 477 (2008) 573

[13] M.S. Pshirkov et al., Astrophys. J. 738 (2011) 192

[14] A. Keivani et al., Astropart. Phys. 61 (2014) 47

[15] G. Giacinti \& D.V. Semikoz, Phys. Rev. D 83 (2011) 083002 